Original Article

\title{
The Intervention Effects of Different Treatments for Chronic Low Back Pain as Assessed by the Thickness of the Musculus Transversus Abdominis
}

\author{
Qiuchen Huang, $\mathrm{PT}^{1-3)^{*}}$, Desheng Li, $\mathrm{PT}^{1-3)}$, Yuying Zhang, $\mathrm{PT}^{1)}$, Gu Rui, $\mathrm{PhD}^{2,3)}$, \\ Ming Huo, PT, $\mathrm{PhD}^{1)}$, Hitoshi Maruyama, PT, $\mathrm{PhD}^{1)}$ \\ 1) Department of Physical Therapy, International University of Health and Welfare: 2600-1 \\ Kitakanemaru, Ohtawara City, Tochigi 324-8501, Japan \\ 2) Department of Physical Therapy, China Rehabilitation Research Center, China \\ 3) School of Rehabilitation Medicine, Capital Medical University, China
}

\begin{abstract}
Purpose] The purpose of this study was to examine the immediate effects of an intervention comprising proprioceptive neuromuscular facilitation (PNF) or neuromuscular joint facilitation (NJF) on chronic low back pain as assessed by the thickness of musculus transversus abdominis [Subjects] The subjects were 12 young people (five males, seven females) who had chronic low back pain on one side for more than 6 months. [Methods] Subjects were asked to lie their sides with the painful side facing up. The subjects received PNF or NJF exercise treatments. The changes in the musculus transversus abdominis thickness were measured using ultrasonography. [Result] The thickness of the musculus transversus abdominis in the NJF group increased significantly and was higher than that in the PNF group and at rest. [Conclusion] The results showed that significantly better improvement can be obtained for chronic low back pain by applying NJF patterns to the musculus transversus abdominis.

Key words: Neuromuscular joint facilitation (NJF), Musculus transversus abdominis, Low back pain
\end{abstract}

(This article was submitted Jan. 24, 2014, and was accepted Mar. 5, 2014)

\section{INTRODUCTION}

Chronic low back pain (LBP) has been considered as a recurring human disease. In order to walk upright and hold that position, humans exert increasing heavy burden on the lumbar region. LBP results from many factors (multifactorial disease), so it cannot be managed by a simple treatment.

The role of stabilization of the multifidus muscle for the lumbar region has been reported. The inner muscles (multifidus muscle and transverse abdominal muscle) are highly correlated with the stability of the lumbar region ${ }^{1)}$. In the case of low activity of inner muscles, outer muscles (erector spinae, musculus rectus abdominis, and abdominal oblique) compensate to maintain keeping the stabilization of the lumbar region. The compensation is one of the causes of LBP.

The muscles of the trunk of the body that participate in maintaining stability can be classified into the global muscle system and local muscle system. Trunk movement is controlled by the global muscle system, which plays a role in supporting the spine and consists of the shallow lumbar muscles. The vertebra is connected by the local muscle sys-

\footnotetext{
*Corresponding author. Qiuchen Huang (E-mail: qiuchen_1984@126.com)

(C)2014 The Society of Physical Therapy Science. Published by IPEC Inc. This is an open-access article distributed under the terms of the Creative Commons Attribution Non-Commercial No Derivatives (by-ncnd) License $<$ http://creativecommons.org/licenses/by-nc-nd/3.0/>.
}

tem which, controls the movement of the lumbar vertebra and consists of the deep lumbar muscles. Although the support provided to the spine is very strong, the symptoms of LBP will still appear ${ }^{2}$, if functional lumbar vertebra movement is lost.

The local muscle system contains many muscles, such as the multifidus muscle, longissimus lumborum, iliocostalis lumborum muscle, medial fiber of the lumbar quadrate muscle, intertransversalis, and interspinal muscles. The internal abdominal oblique muscle (the fibers adhering to the fascia thoracolumbalis) is a constituent of the local muscle system. The stabilization of the lumbar region depends on the multifidus muscle, musculus transversus abdominis, and a part of the oblique abdominal muscle that looks like a ring structure.

The intra-abdominal pressure will be increased by the tonus of the fascia thoracolumbalis, and this is affected simultaneously by the contraction of the musculus transversus abdominis and the multifidus. The increase in fascia thoracolumbalis tonus and the intra-abdominal pressure resulting from the muscle contraction could also contribute to the regional support of each spinal vertebral arch. In particular, support of the sacroiliac joint, one of the structures that are essential to spine stabilization, is affected by the musculus transversus abdominis and the lower fiber of the internal abdominal oblique muscle ${ }^{2}$.

When humans engage in some activities, contraction of the musculus transversus abdominis occurs ahead of the trunk muscle groups ${ }^{3)}$. In preparation for trunk movement, 
the intra-abdominal pressure (IAP) increases.

Recently, rapid progress is being made in research on the pelvic floor muscles. The stabilization of the lumbar region is determined by the inner unit, which is composed of the pelvic floor muscles, musculus transversus abdominis, multifidus muscle, and diaphragm. Active approaches to treatment of urinary incontinence and low back pain have begun to be used in clinical practice.

In clinical research, the resistance movement of the pelvis, the pattern of Proprioceptive Neuromuscular Facilitation (PNF), mobilization is always used in the treatment of LBP, and the static contraction is selected in clinical treatment. Although this treatment relieves symptoms temporarily, the effect does not last long.

NJF is a new therapeutic exercise based on kinesiology that integrates the facilitation element of proprioceptive neuromuscular facilitation and the joint composition movement, aiming to improve the movement of the joint through passive exercise, active exercise, and resistance exercise ${ }^{4)}$. It is used to increase strength, flexibility, and range of motion (ROM).

The aim of this study was to examine the difference in immediate effects of intervention between the PNF and NJF patterns on the pelvis.

\section{SUBJECTS AND METHODS}

The subjects were twelve young people ( 5 males, $7 \mathrm{fe}$ males) who had suffered chronic low back pain for more than 6 months on one side of their body. Subject characteristics are detailed in Table 1. The purpose and contents of this research were explained to the subjects, and all subjects gave their informed consent to participate in the study. The study was approved by the Research Ethics Committee of the International University of Health and Welfare, and the IRD number for this study is $12-155$.

Before the treatment, the subjects were evaluated for pain severity by using a visual analogue scale (VAS). The subjects lay on their sides with the painful side facing up in the resting state and during the two interventions: the front inferior pelvic pattern of PNF and the front inferior pelvic pattern of NJF.

1. PNF group: In the front inferior pelvic pattern of PNF, the two hands of the examiner were placed against the knee which is upside. When the subjects performed the front inferior pelvic pattern, the traction and resistance were applied throughout the process. The static resistance and the traction were applied in the interposition of the PNF pattern by the examiner.

2. NJF group: In the front inferior pelvic pattern of NJF, one hand of the examiner was placed against the knee, and the traction and resistance were increased as in the PNF pattern. The examiner's other hand was placed on spinous process of L3 to prevent the upward curvature. All interventions were carried by one physiotherapist.

The maximum contraction time of 5 seconds was maintained for the intermediate region in every pattern used for the interventions. During this time, the thickness of the musculus transversus abdominis was measured. Each
Table 1. Subject characteristics

\begin{tabular}{lc}
\hline \multicolumn{2}{c}{$\mathrm{M} \pm \mathrm{SD} \mathrm{N}=12$} \\
\hline Age $(\mathrm{y})$ & $24.5 \pm 6.7$ \\
Height $(\mathrm{cm})$ & $168.5 \pm 11.2$ \\
Weight $(\mathrm{kg})$ & $64.3 \pm 12.9$ \\
VAS & $3.3 \pm 1.7$ \\
\hline
\end{tabular}

Table 2. Thickness of the musculus transversus abdominis $(\mathrm{cm})$

\begin{tabular}{lll}
\hline a.Resting & $0.46 \pm 0.04$ & $\mathrm{a}<\mathrm{b}, \mathrm{c}^{*}$ \\
b.PNF & $0.53 \pm 0.05$ & $\mathrm{~b}<\mathrm{c}^{*}$ \\
c.NJF & $0.60 \pm 0.06$ & \\
\hline${ }^{*} \mathrm{p}<0.05$ & &
\end{tabular}

measurement was carried out twice, and the average value was used for analysis. Ultrasound images of the multifidus muscle wall were obtained by using a SonoSite ultrasound system (SonoSite180 PLUS, B mode, $5 \mathrm{MHz}$ linear transducer). Gel was interposed between the transducer and the skin. The transducer was positioned adjacent to and perpendicular to the abdominal wall, $25 \mathrm{~mm}$ anteromedial to the midpoint between the ribs and ilium on the midaxillary line, and parallel to the muscle fibers of the transversus abdominis ${ }^{5}$. The same physical therapist took all measurements to avoid inter-rater errors. Ultrasound images were saved as still images. All thickness measurements were for muscle only, that is, between the fascia boundaries.

In order to determine the min effect of the 2 intervention methods, one-way analysis of variance with the Bonferroni correction was used; the factor was the thickness of the musculus transversus abdominis. The data were analyzed by using SPSS Ver. 17.0 for Windows.

\section{RESULTS}

The results for thickness of the musculus transversus abdominis in the 12 subjects are shown in Table 2.

The thickness of the musculus transversus abdominis increased significantly in the PNF and NJF groups compared in the resting state. The increase in the NJF group was the highest.

\section{DISCUSSION}

The concept of the musculus transversus abdominis being very important for the stabilization of trunk has been recognized by the public, but in clinical fields, research about the contribution of the transversus abdominis has been comparatively ignored.

In the traditional scenario, the therapist would always place special emphasis on a role of the combination therapy composed of LBP exercise and lumbar stabilization exercise.

However, no objective assessment of each traditional method has been performed. In this study, the transverse 
abdominal thickness differed between groups after applying the PNF and NJF techniques. Compared with the transverse abdominal thickness after the PFN technique, that after the NJF technique was significantly increased. The reason for this was that the proximal resistance exerted by the NJF pattern promoted a decrease in transverse abdominal thickness by multifidus muscle contraction and increased intraabdominal pressure.

In recent years, the effects of training using the multifidus and transversus abdominis, like the muscle corset, on LBP have been unclear, and the recurrence rate of LBP is quite high ${ }^{6}$. If the muscles overcompensate, the activities of the multifidus and transversus abdominis will be suppressed. The skill required to contract the multifidus and transverse abdominal without using the global muscles is very difficult to learn. The results of the present study suggest that the control of the musculus transversus abdominis and the entire local muscle system can be improved by closed kinematic chain exercise and localized muscle treat- ment. Clinically, training of the transverse abdominal muscle with the NJF pattern is expected to be used in uroclepsia and LBP for good effects.

Future studies need to investigate the different effects of different treatments after long-term intervention for patients with LBP or urinary incontinence.

\section{REFERENCES}

1) Richardson C: Therapeutic Exercise for Spinal Segmental Stabiliation in Low Back Pain. Edinburgh: Churchill Livingstone, 1999.

2) Bergmark A: Stability of the lumbar spine. A study in mechanical engineering. Acta Orthop Scand Suppl, 1989, 230: 1-54. [Medline] [CrossRef]

3) Richardson C, Jull G, Hodges P: Therapeutic exercise for spinal segmental stabilizationin low back pain. Edinburgh: Churchill Livingstone; 1999.

4) Huo M: Neuromuscular Joint Facilitation. Tokyo: Ipec press, 2010, p 3.

5) Critchley $D$ : Instructing pelvic floor contraction facilitates transversus abdominis thickness increase during low-abdominal hollowing. Physiother Res Int, 2002, 7: 65-75. [Medline] [CrossRef]

6) Hides JA, Jull GA, Richardson CA: Long-term effects of specific stabilizing exercises for first-episode low back pain. Spine, 2001, 26: E243-E248. [Medline] [CrossRef] 